\title{
Contour Lines Generation in Karstic Plateaus for Topographic Maps
}

\author{
Guillaume Touya $^{a,}{ }^{*}$, Hugo Boulze ${ }^{b}$, Anouk Schleich ${ }^{b}$,Hervé Quinquenel $^{c}$ \\ ${ }^{a}$ Univ. Gustave Eiffel, LASTIG GEOVIS, IGN, ENSG, F-94160 Saint-Mande, France, firstname.lastname@ign.fr \\ ${ }^{b}$ ENSG, Univ. Gustave Eiffel, firstname.lastname@ensg.eu \\ ${ }^{c}$ IGN, Department of Cartography, firstname.lastname@ign.fr \\ * Corresponding author
}

\begin{abstract}
Contour lines are a key features of topographic maps as they make the comprehension of terrain more easy. But they are no longer drawn by cartographers, they are mostly automatically derived from digital terrain models. Despite real progress in this automated derivation, some specific terrain landscapes remain incorrectly depicted with such techniques, and this is the case for karstic plateaus full of sinkholes. This paper proposes a specific automated method to derive better contour lines in plateaus, particularly around sinkholes. The process first detects karstic plateaus with many sinkholes, as well as the individual sinkholes. Then, the DTM is smoothed to better reflect the terrain in the plateau and in its surroundings. As a third step, the contour lines around sinkholes are enhanced to draw legible round features that better reflect the real terrain. The process was implemented in a QGIS plugin and tested on a small area with a karstic plateau in the Jura mountain in France, and the cartographers of IGN, the French national mapping agency assessed the results as a great improvement compared to the generic automated process to derive contour lines.
\end{abstract}

Keywords: contour line, digital terrain model, topographic map, map generalization

\section{Introduction}

Contour lines are a key features of topographic maps as they make the comprehension of terrain more easy (Wilkening \& Fabrikant, 2011; Putto et al., 2014). In order to produce topographic maps more quickly, contour lines are no longer drawn by cartographers based on stereoscopic vision, and are rather automatically derived from digital terrain models (DTM). But the map producers do not want to lose too much on quality when saving production time. Past research on mountain cartography proposed automated processes to draw contour lines as close as possible to the ones drawn by cartographers (e.g. Jaara et al., 2011; Kettunen et al., 2017). However, these automated processes do not perform well on very specific terrain morphologies, for instance karstic plateaus full of small sinkholes (Figure 1). The cartographers at IGN France found several problems with the contour lines generated from the DTM in this specific region using the process from Jaara et al. (2011) (Figure 2):

- insignificant details appear, and the contours are not smooth enough;

- the contours sometimes connect several sinkholes making the visual pattern disappear;

- the contours do not reflect the terrain morphology (most sinkholes are not displayed).

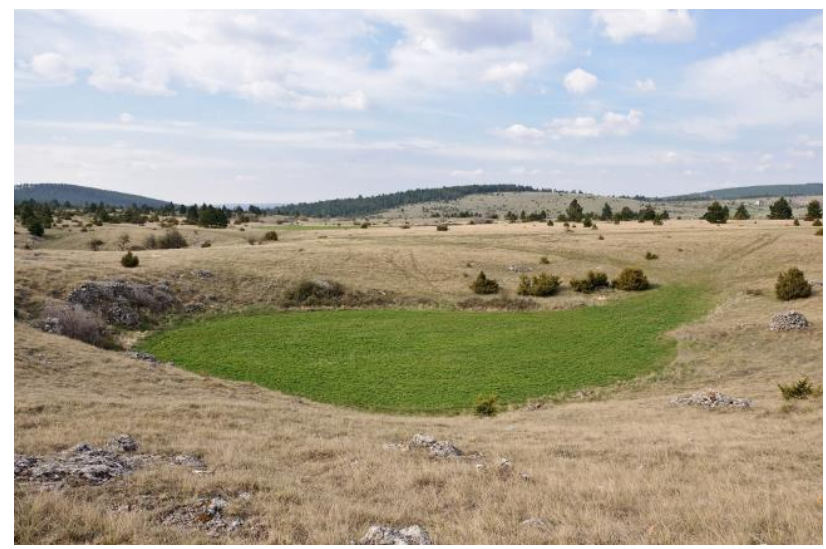

Figure 1. A sinkhole in a karstic plateau in France (CMyrabella / Wikimedia Commons, CC-by-3.0).

These problems could be partly solved with some kind of terrain generalisation that could remove the insignificant details and enhance the significant ones (the sinkholes here) while smoothing everything up. Following the same paradigm as the most advanced research on contour line derivation, i.e. adapting the process to the terrain morphology (Kettunen et al., 2017), there is a clear need for a specific process to improve the contour line derivation from a DTM in this specific kind of regions. This proposes such a specific process that was experimented on the karstic plateaus of the Jura Mountains in France. 


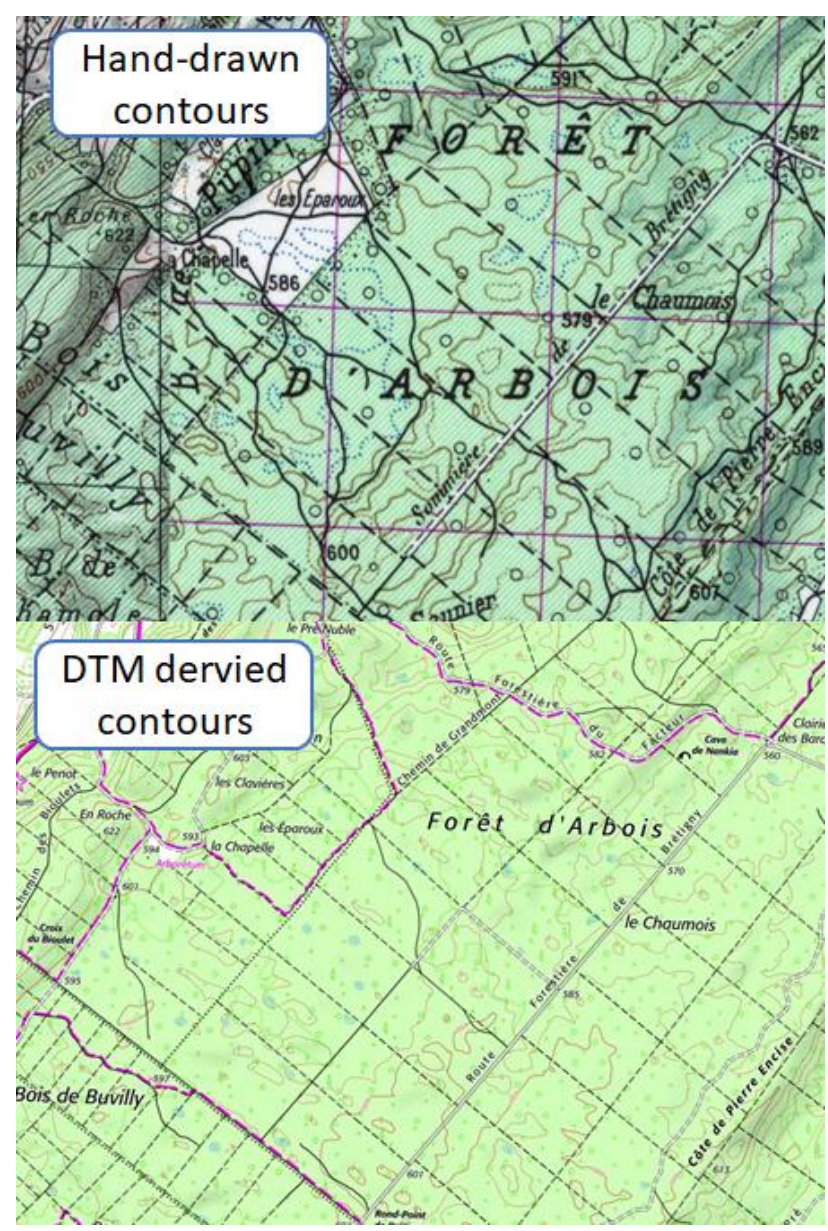

Figure 2. Contour lines manually drawn on the top image, generated from DTM on the bottom image, in a plateau full of sinkholes.

In the second section, related work is presented and discussed. Section 3 describes the complete workflow to automatically derive contour lines in plateaus with sinkholes. Section 4 gives details of implementation and presents results of our experiments. Finally Section 5 draws some conclusions and discusses future research.

\section{Related Work}

Although deriving contour lines from a DTM is straightforward process, the standard techniques available in GIS tools do not give high cartographic quality results. This is why researchers tried to improve the standard techniques, and processing the DTM appeared to be best solution compared to processing the contour lines. The first issue is to characterise the landscape in order to process differently flat and sloping areas. Jaara et al. (2011) separated plains and mountains, and then identified talwegs in both areas. Kettunen et al. used the Topographic Position Index (TPI) to characterise a local terrain. The TPI is a difference between the elevation at one point of the DTM and the mean of its surrounding elevations. The consistency with water bodies, rivers or lakes is also a key issue for relief rendering (Gaffuri, 2007), and the algorithm developed at the US Geological Survey detected the areas where contour lines needed to be consistent with water bodies (Tyler \& Greenlee, 2012). Regarding the transformation of the DTM, two main methods were proposed: an elastic deformation (Jaara et al., 2011) and smoothing (Rosenkranz et al. 2012; Kettunen et al., 2017). Sometimes, the contour lines are also refined after processing the DTM and deriving the contours (Rosenkranz et al. 2012; Tyler \& Greenlee, 2012).

As we plan to remove insignificant details and to enhance the rendering of the underlying structures of the terrain, i.e. the sinkholes, our objectives is very close to a map generalisation problem, and it seems relevant to look at the literature on relief.contour lines generalisation. Guilbert et al. (2014) give a nice overview of these terrain generalisation techniques. Once again, there is clear focus on the classification of landforms to adapt the generalisation operations to these landforms: for instance, Guilbert (2013) identifies the key landforms to derive multi-scale bathymetric contours. But contrary to the papers previously presented on contour line generation, there are many techniques to generalise the contour lines with a removal of symbol overlaps while preserving the shape of terrain (Li \& Sui, 2000; Ai, 2004; Gökgöz \& Selçuk, 2004; Matuk et al., 2006). Such techniques are in fact complementary to the transformation of the DTM.

If we look at the research on automatically deriving hypsometric maps from DTM (Leonowicz \& Jenny, 2011; Samsonov, 2011) or on advanced terrain visualization (Patterson, 2001), the assessment is the same: landform characterisation (thalwegs, peaks, valleys) is a key issue, and smoothing the different landforms gives promising results.

To summarise this section on related work, it is fundamental to first characterise the landforms to choose adapted processes, and then processing the DTM first, and then polish the result with contour line generalisation seems to be the best way to tackle our problem, and the workflow described in the following section follows these principles.

\section{Description of the Workflow}

\subsection{Complete Workflow to Derive Contour Lines}

The proposed workflow to derive high quality contour lines in karstic plateaus with many sinkholes is composed of four steps (Figure 3): (1) globally smoothing the DTM; (2) in parallel with step (1), detect sinkholes individually and the detect the plateaus full of sinkholes; (3) enhance the sinkholes in the smoothed DTM; (4) generate the contour lines from the modified DTMs.These four steps are described in details in the following subsections.

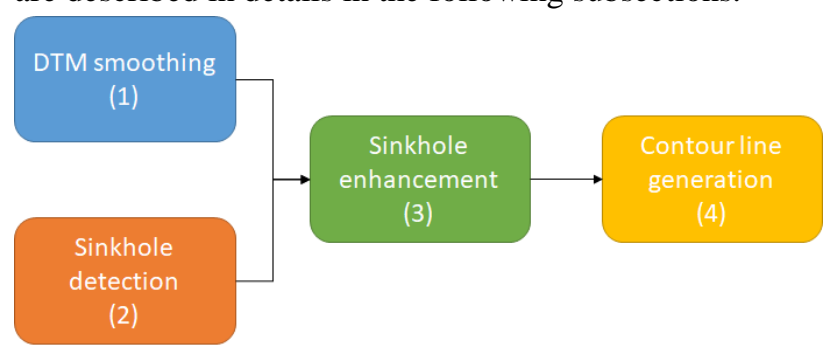

Figure 3. The four main steps of the workflow to derive high quality contour lines in karstic plateaus. 


\subsection{DTM Smoothing}

In order to draw readable contour lines, the original DTM needs to be smoothed. That for we used the method described by Kettunen et al. (2017). The method derives two gaussian smoothings on the initial DTM (DTM1 with a light smoothing, and DTM2 with a strong smoothing). Then the TPI is computed and smoothed (cTPI). Finally we use these three models (DTM1, DTM2, cTPI) to create a new smoothed DTM, called cDTM (Figure4). Equation (1) shows how the models are combined to create cDTM, as proposed by Kettunen et al. (2017).

$$
\begin{array}{r}
c D T M_{i, j}=c T P I_{i, j} * D T M 1_{i, j}+(1 \\
\left.-c T P I_{i, j}\right) * D T M 2_{i, j}
\end{array}
$$

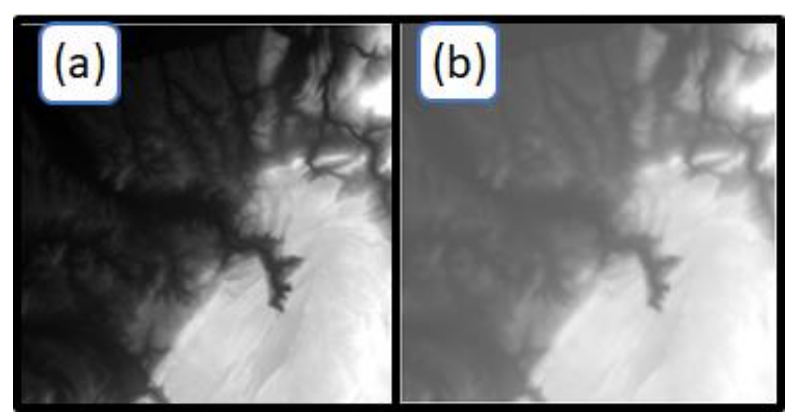

Figure 4. (a) initial DTM. (b) cDTM, the smoothed terrain model.

\subsection{Detection of the Sinkholes}

The aim of this step (2) is to detect all the sinkholes in karstic plateaus, and we need to detect karstic plateaus first. The detection of the sinkholes and the plateaus are both based on the detection of depressions in flat areas. This step (2) is divided into seven sub-steps (Figure 5).

To begin (2.1), we applied a gaussian smoothing to our initial DTM (not the CDTM from step (1)). Then we subtracted the original DTM to this smoothed DTM and if the difference between the two DTMs is higher than 1.5 meters, then we keep these as depressions that are potential sinkhole. Now we have several pixels which were identified by this first detection, but all are not sinkholes, so the following steps are different filters to only keep the depressions that are sinkholes in a karstic plateau.

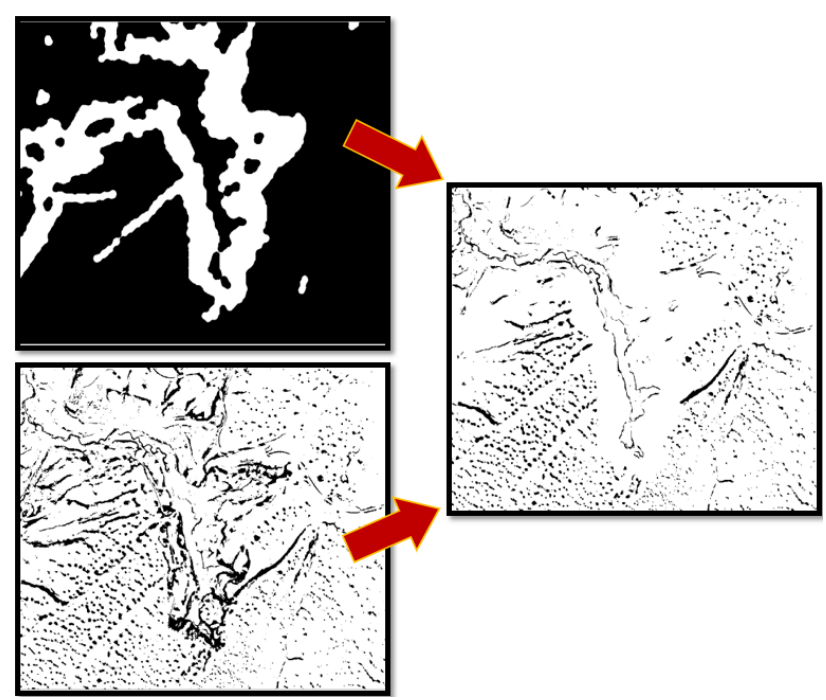

Figure 6. Illustration of steps (2.1) to (2.3) with the flat/sloping mask on the upper left, the difference between DTMs to gives depressions deeper than 1.5 meters, and on the right, the depression located in a flat area.

Then, in step (2.2), we compute a mask of flat/sloping regions using the same principle as Jaara et al. (2011). This mask is used in step (2.3) to keep only the depressions that are located in flat regions. Steps (2.1) to (2.3) are illustrated in Figure 6.

After the application of this mask on our potential sinkholes the raster file is vectorized to process it with vector based measures (2.4). We decided to delete the depressions that are too small and divided the sinkholes on two categories based on their Miller compactness (equation 2). The Miller index is often used to measure the compactness of polygons, particularly to detect round polygons such as roundabouts in a road network (Touya, 2010).

$$
\text { compactness }=\frac{4 \cdot \pi \cdot \text { area }^{\text {perimeter }}}{2}
$$

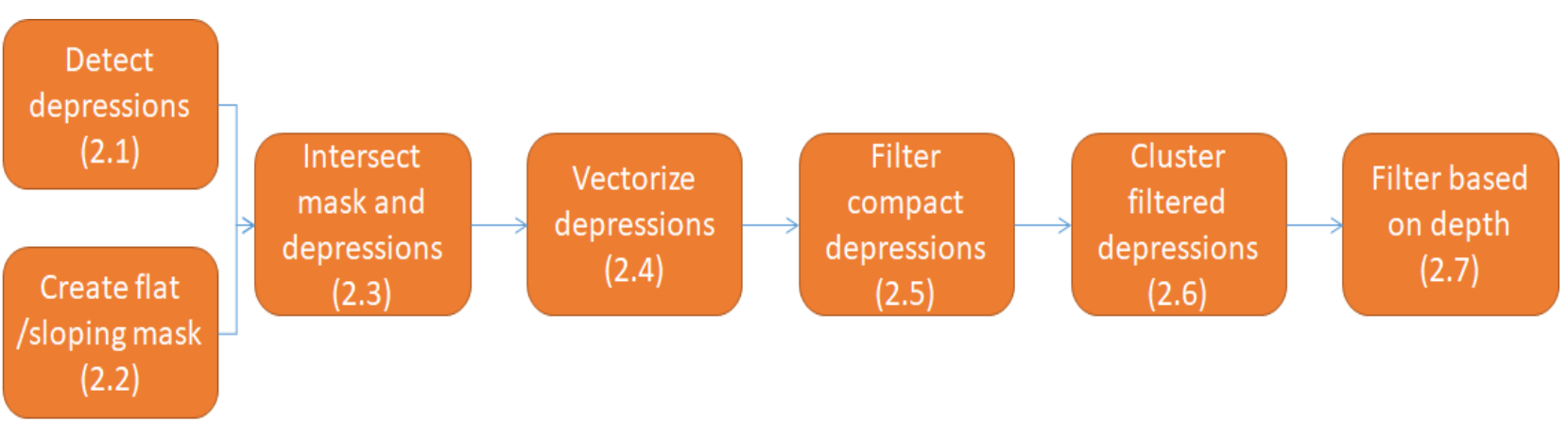

Figure 5. The workflow with the seven steps of the detection of sinkholes. 


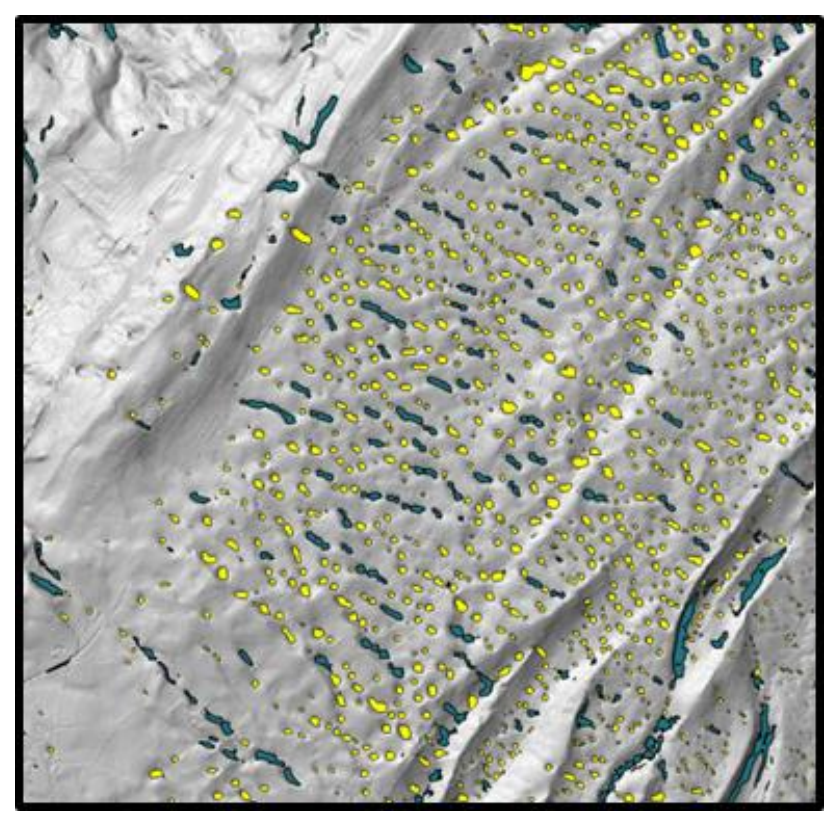

Figure 7. In yellow the round depressions, and in blue the long depressions, under the compactness threshold.

In the step (2.5), the depressions with a compactness index above 0.33 are labeled as round depressions and the depressions with an index below this threshold are labeled as long depressions (Figure 7).

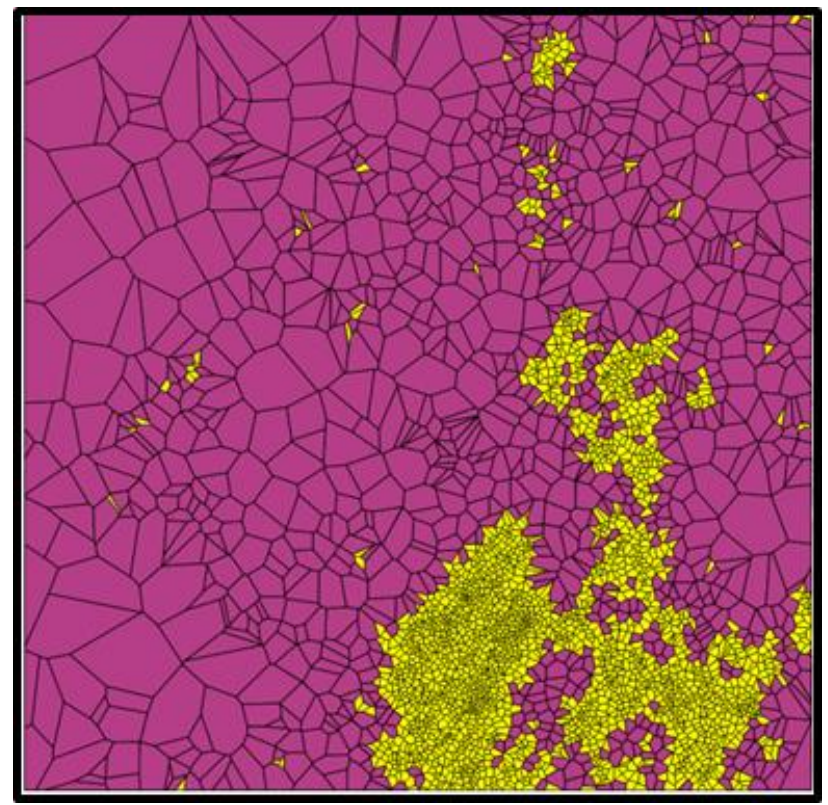

Figure 8. Voronoï based clustering of the depressions (clustered cells in yellow).

Separating round and long depressions is useful as different filters will be used to determine if they are sinkholes or not: the not too long depressions which are in karstic plateaus can be considered as sinkholes, while the round depressions, even if they are not in dense sinkhole regions can also be considered as sinkholes. To identify the dense sinkhole regions, which is the step (2.6), we calculate a Voronoi diagram on the centroids of the round depressions. Then we only keep the regions with a surface smaller than $60000 \mathrm{~m}^{2}$ and merge them (Figure 8). Then we only keep the merged surfaces which cover at least $200000 \mathrm{~m}^{2}$ and apply a small buffer of 100 $\mathrm{m}$. Now that we have identified our dense sinkhole regions we sort out the vectors of long depressions that intersect with the dense region. On these selected depressions, we keep the ones with an area between 700 and $9500 \mathrm{~m}^{2}$ (i.e. the small ones) and a compactness above 0.11 (i.e. the more compact). All the other long depressions are deleted.

Afterward we separate the round sinkholes in the dense zone and the round sinkholes outside of the dense zone. Then, a compactness threshold is fixed higher for the depressions out of the dense regions. We only keep the depressions bigger than $1000 \mathrm{~m}^{2}$ and with a compactness index higher than 0.38 , i.e. only the most significant are kept outside the regions containing many depressions.

At this stage, (2.7), we have three different layers with potential sinkholes which passed our filtering steps: round depressions inside the dense zones, very round and big depressions outside the dense zone, and long depressions inside the dense regions. As we merged the three layers together, we noticed that there were still some false sinkholes. We figured they were often located in thalwegs. To filter out these thalwegs we made a buffer of $15 \mathrm{~m}$ around the sinkholes. The buffer gives us important information about the terrain around the sinkhole and makes sure we include the highest point of the sinkhole to calculate its depth (Figure 9).
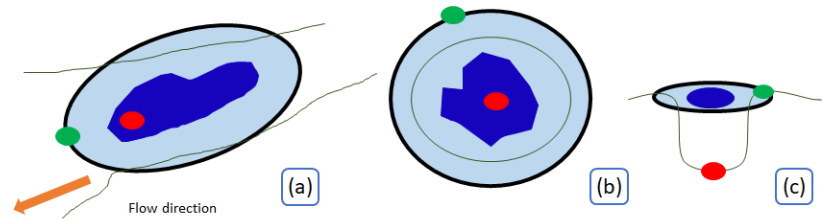

Figure 9. green point is minimal altitude of the outline of the buffer, red point is minimal altitude of detected sinkhole (a) a thalweg; (b) a sinkhole; (c) sectional view of the sinkhole

Then, the outline of this buffer-polygon is extracted and its varying altitude is measured. We then use the minimum altitude of the depression polygon and the minimum altitude of the outline of the buffered polygon to sort out the thalwegs from the sinkholes (Figure 9). If the difference between the minimum altitude of the outline (green point in Figure 9) and the minimum altitude of the depression (red point in Figure 9) is below $0.8 \mathrm{~m}$ then we consider the detected depression as a thalweg and not a sinkhole, and delete it. As we see on the figures in the case of a thalweg the minimum of the altitude of the depression is very close to the minimum altitude of the outline. Contrary to the case where we have a real sinkhole where the sinkhole forms a bowl and the minimum altitude of the sinkhole is much lower than the minimum altitude of the outline. 
At the end of this step (2.7), we have a vector layer containing all the detected sinkholes as well as regions with a high density of sinkholes.

\subsection{Enhancement of the Contour Lines Around Sinkholes}

The next step (3) is to adjust, or caricature the sinkholes. Caricature is a generic operation in map generalisation that exaggerates some characteristics of a map feature to make it more legible. For instance, mountain roads are often caricatured by removing some bends to avoid symbol coalescence (Lecordix et al., 1997). The aim here is to make sure that a contour line will be drawn inside the sinkhole. To dilate the sinkholes we didn't use the approach of morphological filters (dilation/erosion), but implemented a flooding algorithm. The algorithm transforms the detected sinkholes to more realistic morphologies. Consequently the contour lines of the sinkholes will be more accurate. We "fill" the sinkholes until they double their original surface. Once the sinkholes are flooded, we calculate the minimum altitude, the maximum altitude and the depth using the cDTM created at step (1).

If the contour lines of sinkholes do not appear on the topographic maps, it is because they are not located on an altitude at which a contour line is drawn. Most of the times, they are located between two equidistant contour lines (Figure 10). Our idea is to artificially make the sinkhole intersect these contours lines. Therefore we apply a sinkhole adjustment algorithm. It adjusts the sinkholes to the closest contour line. If the sinkhole is closer to the lower equidistant, then the sinkhole is intersected with it (Figure 10, on the right). Otherwise it is intersected with the upper contour line altitude.

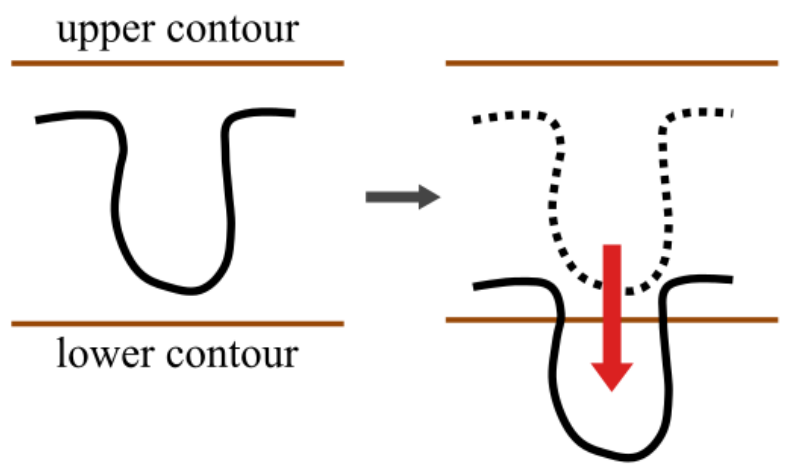

Figure 10. When a sinkhole has maximum and minimum altitudes between two successive contour altitudes, the sinkhole altitude is adjusted to intersect the closer contour line altitude.

However there remains the question of which zone of the sinkhole should intersect with the contour line. It should be intersected in the zone which represents the sinkhole the best. That for we intersect the sinkhole at two different intersection heights, once with a threshold of 3 and once with a threshold of 1,2. These empirical thresholds are noted as $\mathrm{s}$ in equation 3 .
intersectionLevel $=\max ($ altitudes $)-d / s$

where $\mathrm{d}$ is the depth of the sinkhole and $\mathrm{s}=3$ when the sinkhole is symmetric (Figure 11, case 1 ) and $s=1,2$ when the sinkhole is asymmetric (case 2 in Figure 11). To adjust the sinkholes we lower or raise the altitude of the pixels which compose the sinkholes.
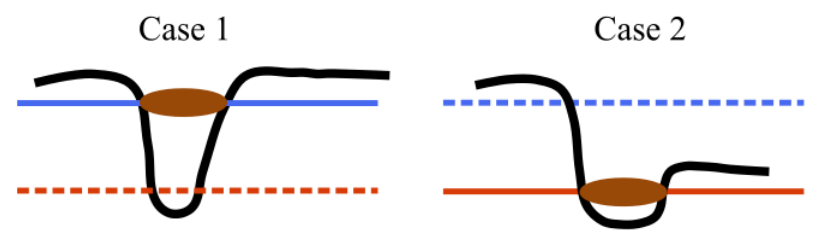

Figure 11. When a sinkhole is symmetric (case 1) the contour line will be drawn on top of it. When the sinkhole is asymmetric (case 2), the contour line is drawn at the bottom of it.

We only treated the case where the sinkholes depth is smaller than the equidistance of the contour lines. We consider that when the sinkholes have a bigger depth than the equidistance they necessarily intersect a contour line, and there is no need to caricature it.

\subsection{Contour Lines Generation}

Finally we generate the contour lines in step (4). First, we generate a first set of contours from the cDTM created at step (1). This will give us the basic smoothed contour lines, but this does not contain the adjusted sinkholes. So we generate new contour lines from the two DTMs created just before in step (3) (i.e. with $s=3$ and $s=1,2$ ). Figure 12 shows the contour lines generated from these two DTMs.

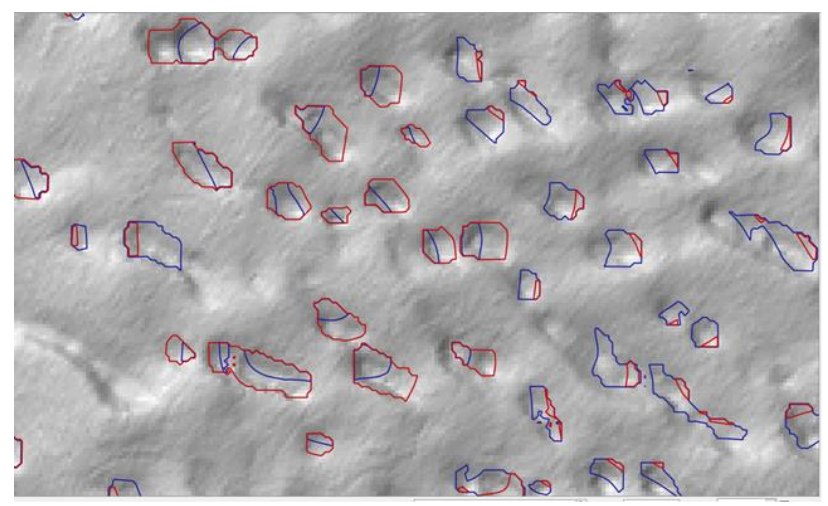

Figure 12. In red the contour lines from the adjustment DTM with $\mathrm{s}=3$ (case 1), and in blue from the adjustment with $\mathrm{s}=1,2$ (case 2$)$.

At this point of the processus we have 3 different layers of contour lines:

- C1: contour lines generated from the cDTM

- C2: contour lines generated at the $\mathrm{s}=3$ threshold

- C3: contour lines generated at the threshold $s=1,2$ 
To merge these three layers, we first remove the contour lines of $\mathrm{S} 2$ and $\mathrm{S} 3$ which are in commun with $\mathrm{S} 1$ as these lines are redundant in all layers.

For each of the sinkholes there are two contour lines: from $\mathrm{C} 2$ and $\mathrm{C} 3$. To choose the one that represents the sinkhole the best, we propose to keep the outer contour line, i.e. the largest one. We can now merge the three layers together and obtain our final result of contour lines of a karstic plateau (and in the neighbouring regions).

\section{Results and Evaluation}

This process was implemented as a QGIS plugin that should be released in the QGIS open source repository by the time of the conference. The DTM used in our experiments was captured from a LIDAR survey, with a 5 $\mathrm{m}$ resolution. The test area is $20,4 \mathrm{~km} \times 20,4 \mathrm{~km}$ wide, near Arbois in the Jura Mountains in France, with some karstic plateaus, plains and valleys. Figure 13 shows some results of the detection of sinkholes in this test area. Figure 13c shows that some sinkholes were filtered out while they could have been retained. The evaluation we carried out showed that $91 \%$ of what we visually assessed as sinkholes were detected, so $9 \%$ are still missing and this part of the process might be improved. Figure 14 shows how many depressions were filtered in step (2) to retain the sinkholes.

The contour lines generated after our complete process are presented in Figure 15. The result is promising as it really captures the essence of the karstic landscape with contour lines. However, the highlighted areas in Figure 15 show some remaining problems:

- when contour lines are rendered with a line symbol, sinkholes close to a "main" contour line may have their symbols intersect;

- some very small sinkholes that should not be rendered at this scale are kept.

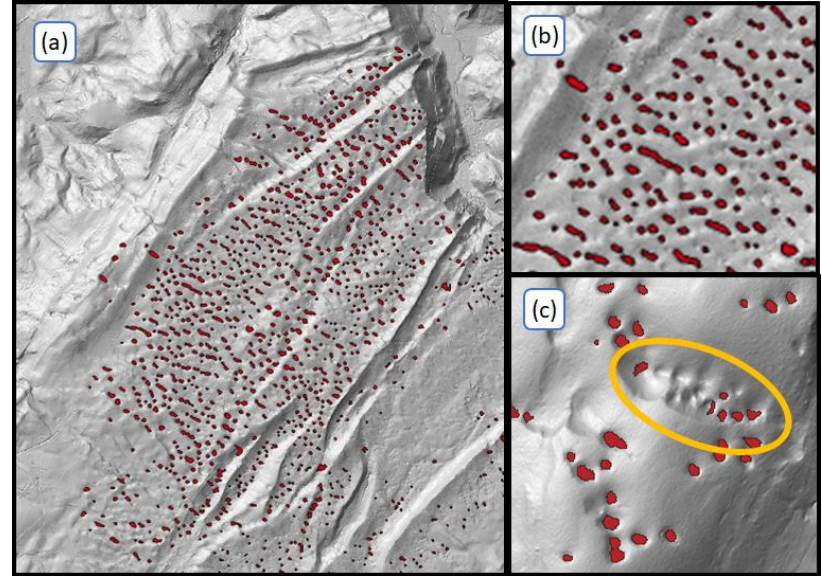

Figure 13. Results of the detection of sinkholes in the Arbois area. (b) shows a zoomed extract and (c) shows an area where some sinkholes are missing.

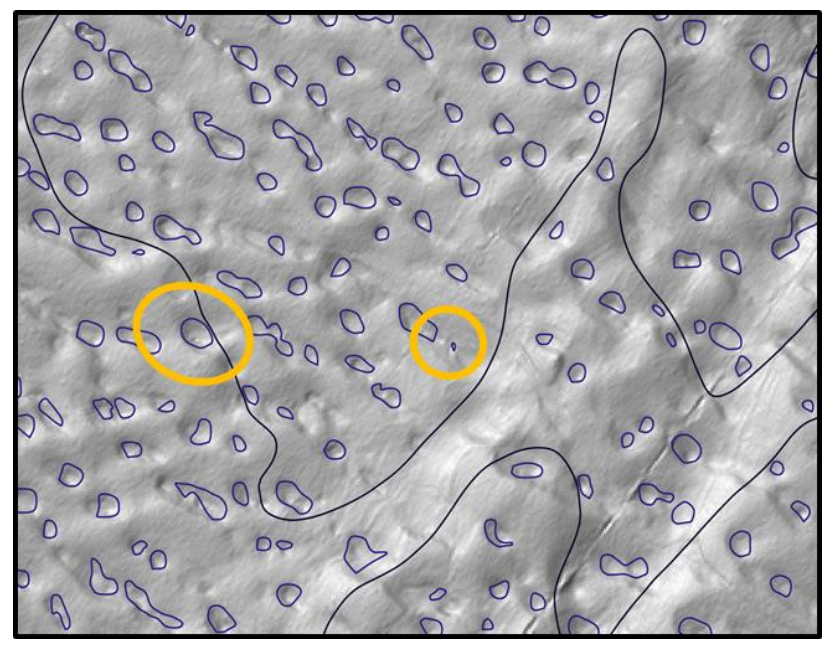

Figure 15. Final contour lines displayed on top of the DTM. The contours in black are the ones from the cDTM and the contours in blue are the ones added with sinkhole adjustment. The circled areas show remaining problems.

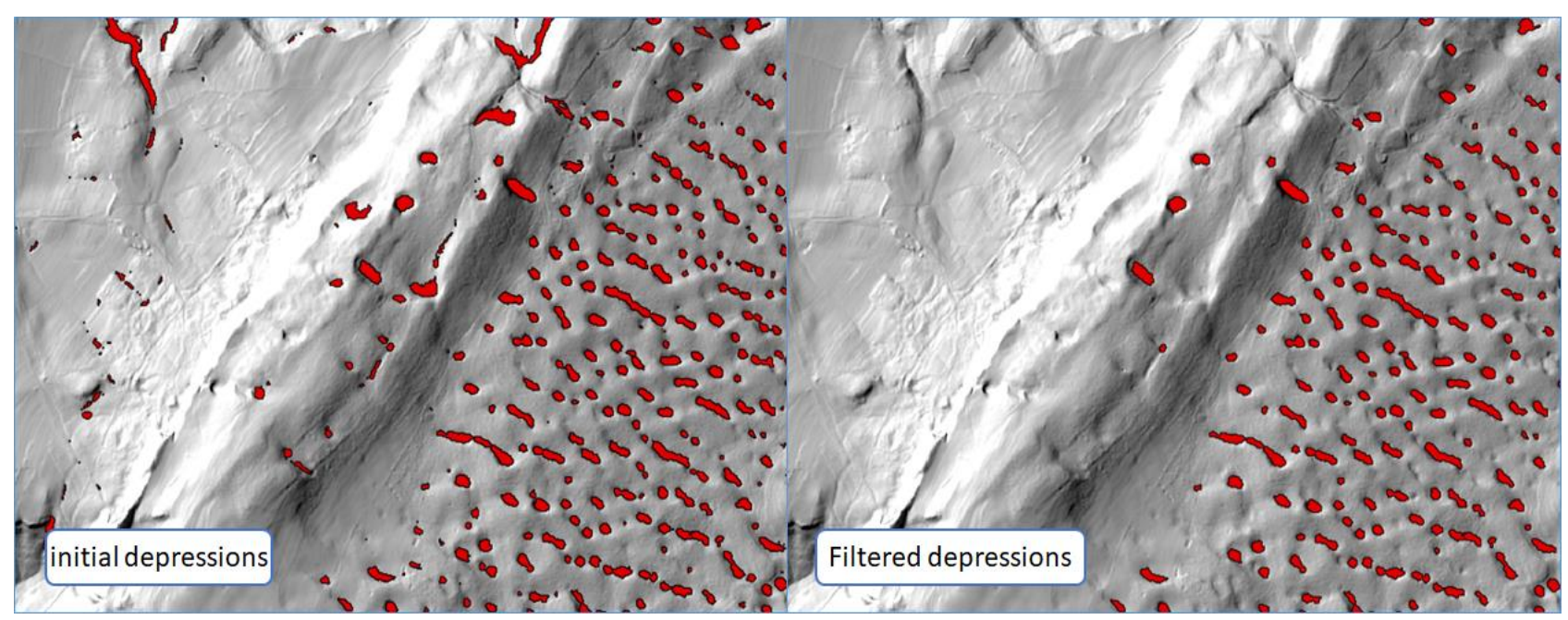

Figure 14. Depressions on top of the DTM before and after the filtering steps. 


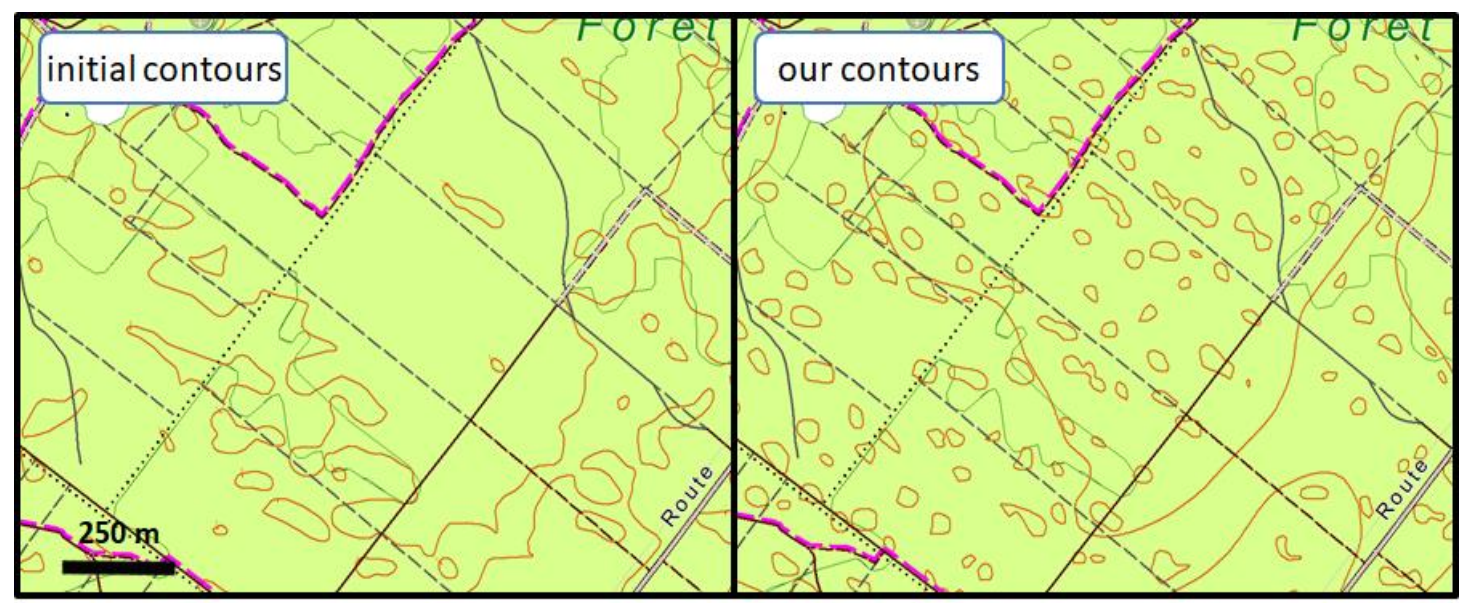

Figure 16. The contour lines obtained with the presented method integrated in the 1:25k topographic map and compared with the current map.

Finally, Figure 16 shows the contour lines generated with the proposed process integrated in a 1:25k topographic map. The result is clearly better than the initial map, and the cartographers from IGN France that evaluated this output confirmed its much higher quality, and they may use our proposition in a near future.

\section{Conclusion and Future Research}

To conclude, we proposed in this paper a new automatic process to generate contour lines from a DTM that goes further than the state-of-the-art in the specialization to specific existing landforms. Such a specialization was called for by cartographers to ensure high quality contour lines even with very specific landscapes. The originality of this approach is the mix of raster- and vector-based techniques, as well as the use of caricature that comes more from map generalization than from terrain rendering techniques.

We have different plans to extend this work. First, the proposed process can be improved in all its steps. For instance, we measured that only $91 \%$ of the areas covered by sinkholes manually captured were automatically detected in step (2). Then, to improve the contour lines derivation, we would like to integrate other generalization operations than caricature. For instance, merging two close sinkholes into one might be sometimes a better operation than enlarging/digging both sinkholes. We also plan to integrate other types of algorithms to polish the contour lines and remove remaining visual conflicts when two lines are too close to each other (Li \& Sui, 2000; Ai, 2004; Gökgöz \& Selçuk, 2004; Matuk et al., 2006). Complementary to generalization, alternative symbolizations could be used to better render karstic plateaus, such as the use of point symbols for small sinkholes. all these terrain representation propositions should then be assessed with user tests to make sure that the user better understand the landforms with the enhanced contour lines.

Furthermore, the process proposed in this paper was developed for 1:25k topographic maps, but now maps are mostly used online in applications allowing to zoom in and out into maps at different scales. Thus, in a similar way to map generalization (Dumont at al., 2017), the contour line generation process should be extended to derive contour lines for multi-scale maps. Finally, we plan to use a similar approach for other specific terrain morphologies, where the generic contour line generation is far from the quality of manually drawn contours. For instance, ruiniform karstic terrains are very similar to terrains with sinkholes in terms of contour lines, except that peaks replace depressions.

\section{References}

Ai, T. (2004). A Generalization of Contour Line Based on the Extraction and Analysis of Drainage System. International Archives of Photogrammetry and Remote Sensing, XXXV (IV/3). ISPRS. http://www.isprs.org/proceedings/XXXV/congress/com m4/papers/549.pdf

Dumont, M., Touya, G., and Duchêne, C. (2017).

Alternative transitions between existing representations in multi-scale maps. Proceedings of the 2017

International Cartographic Conference, Washington, DC, USA. ICA.

Gaffuri, J. (2007). Outflow preservation of the hydrographic network on the relief in map generalisation. 23rd International Cartographic Conference. Moscow, Russia.

Gökgöz, T., and Selçuk, M. (2004). A New Approach for the Simplification of Contours. Cartographica 39(4): 37-44.

Guilbert, E. (2013). Multi-Level Representation of Terrain Features on a Contour Map. GeoInformatica, 17(2):301-324. https://doi.org/10.1007/s10707-012$\underline{0153-\mathrm{Z}}$

Guilbert, E., Gaffuri, J., and Jenny, B. (2014). Terrain Generalisation. In Abstracting Geographic Information in a Data Rich World, edited by D. Burghardt, C. Duchêne, and W. A. Mackaness, 227-258. Lecture Notes in Geoinformation and Cartography. Springer 
International Publishing. https://doi.org/10.1007/978-3319-00203-3_8

Jaara, K., and Lecordix, F. (2011). Extraction of Cartographic Contour Lines Using Digital Terrain Model (DTM). The Cartographic Journal, 48(2): 131137. https://doi.org/10.1179/1743277411Y.0000000011.

Kettunen, P., Koski, C., and Oksanen, J. (2017). A Design of Contour Generation for Topographic Maps with Adaptive DEM Smoothing. International Journal of Cartography 3(1): 19-30.

https://doi.org/10.1080/23729333.2017.1300998.

Lecordix, F., Plazanet, C., and Lagrange, J.-P. (1997). A Platform for Research in Generalization: Application to Caricature. GeoInformatica 1(2):161-182. https://doi.org/10.1023/A:1009736628698

Leonowicz, A., and Jenny, B. (2011). Generalizing Digital Elevation Models for Small Scale Hypsometric Tinting. Proceedings of 25th International Cartographic Conference. Paris, France.

Li, Z., and Sui, H. (2000). An Integrated Technique for Automated Generalization of Contour Maps. The Cartographic Journal, 37( 1):29-37.

Matuk, K., Gold, C., and Li, Z. (2006). Skeleton Based Contour Line Generalization, 12th International Symposium on Spatial Data Handling, 643-658. Berlin, Heidelberg: Springer-Verlag.

https://doi.org/10.1007/3-540-35589-8_41.

Patterson, T. (2001). DEM Manipulation and 3-D Terrain Visualization: Techniques Used by the U.S. National Park Service. Cartographica, 38(1-2): 89-101.

Putto, K., Kettunen, P., Torniainen, J., Krause, C. M., and Sarjakoski, L. T.. (2014). Effects of Cartographic Elevation Visualizations and Map-Reading Tasks on Eye Movements. The Cartographic Journal 51(3): 22536. https://doi.org/10.1179/1743277414Y.0000000087.

Rosenkranz, B., Knudsen, T., Mortensen, H. E., \& Michealsen, P. B. (2012). Automatic generation of contour lines based on DK-DEM (Tech. Rep. No. 13). National Survey and Cadastre - Denmark. ftp://ftp.kms.dk/download/Technical_Reports/KMS_Te chnical_Report_13.pdf

Samsonov, T. (2011). Multiscale Hypsometric Mapping. In Advances in Cartography and GIScience, edited by A. Ruas, 1:497-520. Lecture Notes in Geoinformation and Cartography. Berlin, Heidelberg: Springer. https://doi.org/10.1007/978-3-642-19143-5_28.

Touya, G. (2010). A Road Network Selection Process Based on Data Enrichment and Structure Detection. Transactions in GIS 14(5):595-614. https://doi.org/10.1111/j.1467-9671.2010.01215.x.

Tyler, D. J., \& Greenlee, S. K. (2012). Creation of digital contours that approach the characteristics of cartographic contours (Tech. Rep. No. 2012-5167). U.S. Geological Survey.

https://pubs.usgs.gov/sir/2012/5167/sir2012-5167.pdf
Wilkening, J., and Fabrikant, S. I. (2011). How Do Decision Time and Realism Affect Map-Based Decision Making? In Spatial Information Theory, edited by M. Egenhofer, N. Giudice, R. Moratz, and M. Worboys, 119. Lecture Notes in Computer Science. Springer Berlin Heidelberg, 2011. 